\section{First-line therapy in chronic lymphocytic leukemia: a Swedish nation-wide real-world study on 1053 consecutive patients treated between 2007 and 2013}

\author{
Sandra Eketorp Sylvan, ${ }^{1}$ Anna Asklid, ${ }^{1,2}$ Hemming Johansson, ${ }^{1}$ \\ Jenny Klintman, ${ }^{3,4}$ Jenny Bjellvi, ${ }^{5}$ Staffan Tolvgård, ${ }^{6}$ Eva Kimby, ${ }^{7}$ \\ Stefan Norin, ${ }^{7}$ Per-Ola Andersson, ${ }^{8}$ Claes Karlsson, ${ }^{1,9}$ Karin Karlsson, ${ }^{3}$ \\ Birgitta Lauri, ${ }^{10}$ Mattias Mattsson, ${ }^{11}$ Anna Bergendahl Sandstedt, ${ }^{12}$ \\ Maria Strandberg, ${ }^{13}$ Anders Österborg, ${ }^{1,9^{*}}$ and Lotta Hansson ${ }^{1,9 *}$
}

${ }^{1}$ Department of Oncology-Pathology, Karolinska Institutet, Stockholm; ${ }^{2}$ Department of Oncology, Karolinska University Hospital, Stockholm; ${ }^{3}$ Department of Hematology, Oncology and Radiation Physics, Skåne University Hospital, Lund; ${ }^{4}$ Department of Translational Medicine, Lund University; ${ }^{5}$ Department of Hematology, Sahlgrenska University Hospital, Gothenburg; ${ }^{\circ}$ Department of Internal Medicine, Östersunds Hospital; ${ }^{7}$ Department of Internal Medicine Huddinge, Karolinska Institutet, Stockholm; ${ }^{8}$ Department of Hematology, South Älvsborg Hospital, Borås; ' Department of Hematology, Karolinska University Hospital, Stockholm; ${ }^{10}$ Department of Hematology, Sunderby Hospital, Sunderbyn-Luleå; ${ }^{11}$ Department of Hematology, Uppsala University Hospital; ${ }^{12}$ Department of Hematology, Linköping University Hospital and ${ }^{13}$ Department of Medicine, Sundsvall Hospital, Sweden

${ }^{\star} A O$ and $L H$ are co-senior authors.

\author{
Haematologica 2019 \\ Volume 104(4):797-804
}

\section{ABSTRACT}

1 he aim of this study was to investigate long-term outcome following first-line therapy in consecutive chronic lymphocytic leukemia (CLL) patients in a well-defined geographic area: Sweden. All patients diagnosed with CLL (2007-2013) ( $n=3672$ ) were identified from national registries, screening of patient files identified all $(100 \%)$ treated first line $(n=1053)$ and for those, an in-depth analysis was performed. End points were overall response rate, progression-free survival (PFS), overall survival (OS), and safety. Median age was 71 years; $53 \%$ had Rai stage III-IV and $97 \%$ had performance status grade $0-2$. Fluorescence in situ hybridization (FISH) was performed in $57 \%$ of patients: $15 \%$ had del(17p). Chlorambucil + prednisone was used in 39\% $(5 \%$ also received rituximab). Fludarabine+cyclophosphamide+rituximab or fludarabine+cyclophosphamide was used in $43 \%$ and bendamustine + rituximab in $6 \%$. Overall response rate was $64 \%$; chlorambucil $43 \%$, fludarabine+cyclophosphamide+rituximab $84 \%$, fludarabine+cyclophosphamide $75 \%$ and bendamustine + rituximab $75 \%$. Median PFS and OS was 24 and 58 months, respectively, both were significantly associated (multivariate analysis) with type of treatment, del(17p), performance status, gender, age and geographical region (OS only). Chlorambucil-treated patients had a median PFS and OS of only 9 and 33 months, respectively. Chlorambucil usage declined gradually throughout the study period, but one-third of patients still received chlorambucil + rituximab in 2013. Infections $\geq$ grade III were significantly associated with treatment; chlorambucil $19 \%$ versus fludarabine+cyclophosphamide+rituximab 30\%. Richter transformation occurred in $5.5 \%$ of the patients, equally distributed across therapies. This is the largest retrospective, real-world cohort of consecutive first-line treated CLL patients with a complete follow up. In elderly patients, an unmet need for more effective, well-tolerated therapies was identified.

\section{Correspondence:}

LOTTA HANSSON

lotta.hansson@sll.se

Received: June 21, 2018.

Accepted: November 19, 2018.

Pre-published: November 22, 2018.

doi:10.3324/haematol.2018.200204

Check the online version for the most updated information on this article, online supplements, and information on authorship \& disclosures: www.haematologica.org/content/104/4/797

(C)2019 Ferrata Storti Foundation

Material published in Haematologica is covered by copyright. All rights are reserved to the Ferrata Storti Foundation. Use of published material is allowed under the following terms and conditions:

https://creativecommons.org/licenses/by-nc/4.0/legalcode. Copies of published material are allowed for personal or internal use. Sharing published material for non-commercial purposes is subject to the following conditions:

https://creativecommons. org//icenses/by-nc/4.0/legalcode, sect. 3. Reproducing and sharing published material for commercial purposes is not allowed without permission in writing from the publisher. 


\section{Introduction}

Chronic lymphocytic leukemia (CLL) is the most common leukemia in the Western world. In Sweden, the incidence is approximately 500 patients per year. The median age at diagnosis is approximately 70 years ${ }^{1-4}$ The clinical course is extremely heterogeneous. At diagnosis, most patients are asymptomatic and the disease may be indolent for a long time. However, many patients show disease progression after a few years. When the disease requires treatment, strategies should be individualized. ${ }^{5}$ Chemoimmunotherapy with fludarabine in combination with cyclophosphamide and rituximab (FCR) resulted in an overall response rate (ORR) of about $90 \%$ and improved overall survival (OS), ${ }^{6}$ and represents the standard treatment in fit patients younger than 65 years. Yet, FCR is less well tolerated in patients over 65 years $^{7}$ and these patients may instead benefit from bendamustine in combination with rituximab (BR) which has shown response rates similar to those achieved with FCR but with less toxicity, ${ }^{8,9}$ For elderly fragile patients, chlorambucil in combination with a CD20 monoclonal antibody ${ }^{10,11}$ could be an alternative; whether BR is to be preferred in old unfit patients remains uncertain. ${ }^{12}$

The presence of TP53 aberrations [del(17p) or TP53 mutation] is strongly associated with chemotherapy refractoriness, early relapse ${ }^{13,14}$ and, until recently, a very dismal prognosis. ${ }^{14-16}$ Hence, evaluation of TP53 status is strongly recommended before treatment initiation. Brutons's tyrosine kinase (BTK) inhibitor ibrutinib ${ }^{17-19}$ has offered new options both for these patients and for relapsed/refractory $\mathrm{CLL},{ }^{20}$ with an ORR of $85 \%$ reported, ${ }^{17,18}$ and is considered the best available option for patients with TP53 disruptions. ${ }^{3,20,21}$ New treatments are costly and frequently accepted by regulatory agencies based on trials conducted in selected groups of patients with PFS, not OS, as end point. Hence, long-term results, including OS estimates, in real-life treated patients are important to determine the optimal therapy for patients with CLL. ${ }^{22}$

Previous data from the United States ${ }^{23}$ suggest that type of area (rural or urban) and type of hospital may influence response and survival especially in patients with high-risk CLL. However, Swedish results may differ due to the fact that almost all patients are treated within public health care. This means that most treatment decisions are taken at therapy conferences and we have a widespread usage of yearly up-dated national CLL guidelines. ${ }^{3}$

The Swedish Cancer Registry and the Swedish National CLL-Registry give us a unique opportunity to identify all patients diagnosed with CLL for an in-depth analysis of every single patient file. This provides a complete record of all patients treated within a defined time period on a nation-wide basis. Thus, this study provides high-quality real-world results on CLL first-line treatment that may be used as quality assurance and may help to interpret the cost-effectiveness of new drugs for healthcare providers. It may also serve as a control for clinical trials, selecting patients based on inclusion/exclusion criteria. Given this, the aim of this study was to investigate the outcome following first-line therapy in a well-defined population of consecutive CLL patients, in a setting with complete follow up.

\section{Methods}

This was a retrospective observational study. All patients diagnosed with CLL according to the World Health Organization criteria from 2007 to 2013 were identified from the National Cancer Registry. A representative physician from each of Sweden's six health-care regions reviewed all the patient files in the region to identify patients who had received first-line CLL treatment due to progressive, symptomatic CLL. Patients who had started therapy before the end of 2013 were included in order to obtain sufficient follow up. Their files were analyzed in detail from the date of diagnosis until death or until the end of the study period (2017), whichever came first. Patients who had only received treatment for autoimmune hemolysis or idiopathic thrombocytopenic purpura (ITP) not related to progressive CLL were excluded. As this was a retrospective observational study, ethics committee approval (2013/952-31/3) was obtained; in Sweden no informed patient consent was required. The study was performed in accordance with the ethical principles of the Declaration of Helsinki ${ }^{24}$ and in compliance with national laws.

\section{Data acquisition and study procedure}

Data on patients' characteristics, treatment, outcome and toxicity were recorded on case record forms (CRFs). Information on participation in clinical trials, type of hospital (county/rural, regional or university) where the main body of treatment was given, where the decision on treatment was taken, geographical region, and whether choice of first-line therapy was compliant to the actual Swedish national CLL guidelines ${ }^{3}$ was also recorded. Furthermore, concomitant medication with acetylsalicylic acid (ASA) or statins were recorded since these drugs appear to induce apoptosis on CLL cells ${ }^{25,26}$ and may improve outcome in FCR-treated CLL. ${ }^{27}$ Data were incorporated in a specially developed version of the Information Network for Cancer Care (INCA) database and systematically cross-checked and validated for accuracy. Treatment response was evaluated according to the 2008 International Workshop on Chronic Lymphocytic Leukemia (IWCLL) criteria. ${ }^{28}$ Major infections (grade III-V) and other serious adverse events (SAE) according to the NCI CTCAE 3.0 were recorded. Richter transformation (RT) and secondary tumors were also recorded. The Swedish Cause of Death Registry was used to validate records of death. ${ }^{29}$

\section{Statistical analysis}

End points in this study were evaluated according to the IWCLL criteria ${ }^{28}$ and included: ORR, duration of response (DOR), PFS, OS and safety. In the analysis of PFS, time was calculated from the start date of first-line therapy to the date of progression or date of death, whichever came first. In the analysis of OS, time was calculated from the date of first-line therapy to the date of death. For event-free patients, time was calculated to the date of last clinical visit.

The Kaplan-Meier method was used to estimate and graphically display OS and PFS. Proportional hazards regression was used to estimate the effect of risk factors on time to failure. Results from these models are presented as hazard ratios (HR) together with $95 \%$ confidence intervals (CI). Reported $P$-values from these models refer to Wald tests.

As FISH analysis was not implemented in the national guidelines until 2010, and cytogenetic status is a strong prognostic and predictive marker, ${ }^{14-16}$ patients were grouped into an earlier treatment period (2007-2009) and a later period (2010-2013). Multivariate analyses were restricted to the latter cohort. Analysis of the impact of IGHV mutation status did not provide 
sufficient power for this time period (only a small fraction of patients was tested); this was, therefore, excluded from the multivariate analysis. However, regarding IGHV, the prognostic impact of the whole population was analyzed separately. Patients who had received allogeneic stem cell transplant after first-line therapy $(n=2)$ were excluded from PFS and OS analysis.

\section{Results}

In total, 3672 patients diagnosed with CLL between 2007 and 2013 were identified from the Swedish Cancer registry for whom all $(100 \%)$ medical files were available for review. Out of these, 1053 patients had started firstline treatment between 2007 and 2013, thus being subject to further in-depth analysis. The six geographical regions included $10-23 \%$ of the patients each. Sparsely populated areas included fewer patients $(10 \%)$ than those with the larger cities. Median follow up for all patients was 4.8 years.

\section{Baseline patients' characteristics}

Baseline characteristics at start of first-line treatment are shown in Table 1. Median age at first-line treatment was 71 years (range 31-96 years). Thirty-four percent were females and the majority (53\%) had advanced disease with Rai stages III-IV. Patients were generally in a good performance status with $97 \%$ in Eastern Co-operative Oncology Group (ECOG) grade $0-2$. In total, results of cytogenetic assessment were available for 599 patients $(57 \%)$; more results were available in the latter time period (64\% 2010-2013 vs. 47\% 2007-2009). Since 2010, when FISH was generally recommended in the national guidelines, there has been a significant difference in the frequency of cytogenetic analysis between the regions (50.8-72.5\%; $P=0.003)$ and cytogenetic analysis has been more often performed at university hospitals $(80 \%)$ than other types of hospitals $(55-60 \%)(P<0.001)$. The older the patient, the more rarely was the analysis performed. In the younger patient group $[<65$ years $(y)]$, cytogenetic analysis was available in $87 \%$ compared to $75 \%$ and $39 \%$ in the middle aged (65-74 y) and oldest ( $\geq 75 \mathrm{y}$ ) groups, respectively. The frequency of $\operatorname{del}(17 \mathrm{p})$ was $4 \%$ and $11 \%$ in the earlier and later time periods, respectively, out of all patients tested $(\mathrm{n}=599)$.

Analysis of IGHV mutational status was, and is still, optional according to the Swedish guidelines and thus was analyzed only in a minority of patients $(n=224$; 20\%): $12 \%$ were unmutated and $8 \%$ mutated.

\section{Treatment}

The majority $(68 \%)$ of patients started first-line treatment in the later time period (2010-2013). Most patients $(63 \%)$ were treated at County/Rural hospitals (i.e. neither university nor regional hospitals), whereas $32 \%$ received their treatment at university hospitals. In almost all cases, the treatment decision was taken at the same institution as that in which the patient was subsequently treated. First-line treatments are shown in Table 2. Type of treatment was unknown in 4 patients and in 4 could not be evaluated. The most frequently used regimens were: chlorambucil (CLB/CLB-R) (39\%), FCR (27\%) and FC $(16 \%)$. Nearly all patients $(95 \%)$ receiving CLB did not receive rituximab. Only $6 \%$ of the patients received bendamustin (B) or (BR) and almost all were treated in the later study period. For all regimens, dosing intensity was similar across geographical regions and type of institution. Treatment was given according to the national guidelines in $80 \%(n=843)$ of the patients, $5 \%(n=49)$ were included in clinical trials, and in $15 \%(n=153)$ the treatment was individual, i.e. neither according to guidelines nor to a clinical protocol. The median age was higher for patients receiving chlorambucil (79 y) and younger for patients receiving FCR (64 y) compared to other chemotherapy-based regimens (F/FC $68 \mathrm{y}, \mathrm{B} / \mathrm{BR} 72 \mathrm{y}$, $\mathrm{CHOP} / \mathrm{CVP}+/-\mathrm{R} 71 \mathrm{y}$ ). The median age in the CLB group did not change over the study period. In patients aged 75 $y$ or over, $73 \%$ received CLB, F/FC (9\%) and B/BR (5\%), whereas in patients under 65 years of age, the most commonly used treatment was FCR (53\%) followed by F/FC $(22 \%)$. Those who received CLB also generally had a worse performance status, with $20 \%$ in ECOG 2-3 compared to $4 \%$ and $5 \%$ of those receiving FCR and BR, respectively. Notably, university hospitals used CLB significantly $(P=0.01)$ less often $(30 \%)$ than other types of

Table 1. Baseline characteristics at start of first-line therapy ( $n=1053)$.

\begin{tabular}{lc} 
Factor & N (\%) \\
Age, median [range] & 71 years [31-96] \\
Male & $691(66)$ \\
\hline ECOG performance status & \\
$0-1$ & $916(87)$ \\
2 & $102(10)$ \\
3 & $19(2)$ \\
Missing & $16(2)$ \\
Binet stage & \\
A-B & $520(49)$ \\
C & $499(47)$ \\
Missing & $34(3)$
\end{tabular}

\section{Rai stage}

0-II 474 (45)

III-IV $\quad 556(53)$

Missing 23 (2)

FISH

\begin{tabular}{lc} 
del(13q) & $198(19)$ \\
Normal & $112(11)$ \\
Trisomy 12 & $103(10)$ \\
del(11q) & $94(9)$ \\
del(17p) & $92(9)$ \\
Missing & $454(43)$ \\
\hline
\end{tabular}

Hospital type

University $341(32)$

Regional 51 (5)

County/Rural $\quad 660(63)$

Missing $1(0)$

Treatment

Guidelines $\quad 843(80)$

Clinical trial $49(5)$

Individual $153(15)$

Unknown 8 (1)

n/N: number; ECOG: Eastern Co-operative Oncology Group; FISH: fluorescence in situ hybridization. 
Table 2. First-line treatment by time period and response/infection rates.

\begin{tabular}{|c|c|c|c|c|c|}
\hline Ireatment & $2007-2009$ & $\begin{array}{c}\text { Time period ( } \%) \\
2010-2013\end{array}$ & $2007-2013$ & $\begin{array}{l}\text { ORR } \\
(\%)\end{array}$ & $\begin{array}{c}\text { Infection" }^{*} \\
\text { > Grade III (\%) }\end{array}$ \\
\hline $\mathrm{F} / \mathrm{FC}$ & $95(29)$ & $74(10)$ & $169(16)$ & $127(75)$ & $48(29)$ \\
\hline FCR & $32(10)$ & $253(35)$ & 285 (27) & $240(84)$ & $84(30)$ \\
\hline Alemtuzumab & $17(5)$ & $31(4)$ & $48(5)$ & $32(67)$ & $17(35)$ \\
\hline $\mathrm{CLB} \pm \mathrm{R}$ & $159(48)$ & $245(34)$ & $404(39)$ & $174(43)$ & $77(19)$ \\
\hline $\mathrm{B} / \mathrm{BR}$ & $1(0)$ & $61(9)$ & $62(6)$ & $47(75)$ & $20(32)$ \\
\hline $\mathrm{CHOP} / \mathrm{CVP} \pm \mathrm{R}$ & $16(5)$ & $11(2)$ & $27(3)$ & $22(82)$ & $9(33)$ \\
\hline Other & $6(2)$ & $26(4)$ & $32(3)$ & $22(67)$ & $8(25)$ \\
\hline Rituximab alone & $4(1)$ & $14(2)$ & $18(2)$ & $9(50)$ & $2(11)$ \\
\hline Total & $330(100)$ & $715(100)$ & 1045 & $673(64)$ & $265(25)$ \\
\hline
\end{tabular}

"For the whole time period 2007-2013.ORR: overall response rate; F: fludarabine; FC: fludarabine in combination with cyclophosphamide; FCR: fludarabine in combination with cyclophosphamide and rituximab; $\mathrm{CLB} \pm \mathrm{R}$ : chlorambucil and rituximab; B/BR: bendamustine/bendamustin and rituximab; $\mathrm{CHOP} / \mathrm{CVP} \pm \mathrm{R}$ : cyclophosphamide+hydroxydaunorubicin+vincristine+prednisone / cyclophosphamide+ vincristine+prednisone+rituximab.

hospitals (43\%). The use of CLB declined significantly over the years, with $58 \%$ usage in 2007 to $31 \%$ in 2013 $(P<0.0001)$. However, by the end of 2013, the CLB firstline usage was still high, varying between regions from $27 \%$ to $49 \%$.

\section{Response to first-line therapy}

The ORR for the study group was $64 \%$ ( $15 \%$ CR): CLB $43 \%$, FCR $84 \%$, FC $75 \%$ and B/BR $75 \%$. ORR was significantly associated with type of treatment $(P<0.001)$, performance status $(P<0.001)$, del $(17 p) \quad(P=0.007)$, age $(P<0.001)$, and compliance to national guidelines $(P=0.003)$, but not with gender, Rai stage or type of hospital (univariate analysis). Patients included in clinical trials showed a numerically better response rate ( $82 \%$ ) than those treated according to national guidelines $(65 \%)$ and compared to patients treated neither according to guidelines nor to a clinical study protocol (54\%).

\section{Progression-free and overall survival}

Median PFS was 24 months (range 20-26 months) and median OS was 58 months (range 40-76 months). At 5-y follow up, $51 \%$ of all patients were deceased, and nearly two-thirds had died from CLL or CLL-related infections.
Progression-free survival in relation to type of first-line therapy is shown in Figure 1A. As expected, the longest PFS was observed with FCR, whereas the median PFS in patients who received CLB was only nine months. PFS in relation to FISH results are shown in Figure 1B. Shortest PFS was observed for del(17p). Similar results were observed for type of treatment and FISH data in relation to OS (Figure 2A and B). Notably, CLB-treated patients had a median OS of only 33 months. Survival in relation to type of hospital is shown in Figure $2 \mathrm{C}$ and in relation to age in Figure 2D.

In a multivariate analysis, both PFS and OS were significantly associated with type of treatment, cytogenetic status, performance status, gender and age (Table 3). OS was also significantly associated with geographical region $(P=0.003)$. There was a tendency but no significant difference $(P=0.07)$ in OS between the two time periods (20072009 vs. 2010-2013). IGHV analysis was only performed in a small fraction of patients and did not provide sufficient power when included in the model (both PFS and OS were non-significant). Thus, this analysis was excluded from multivariate analysis regarding this time period. However, we also analyzed the prognostic impact of IGHV on the whole study population. The results from
A

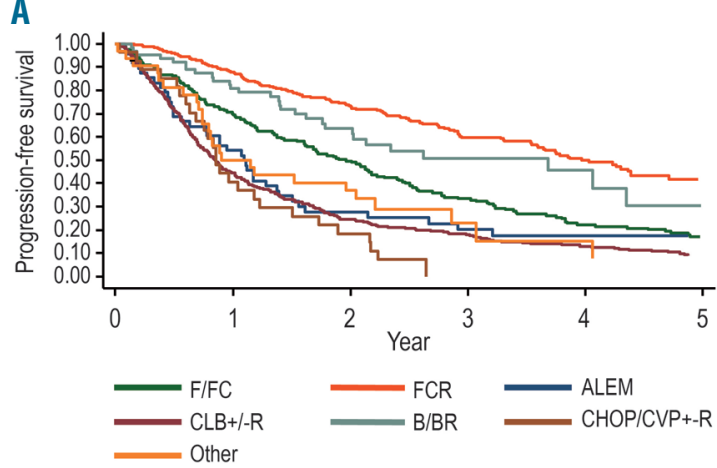

B

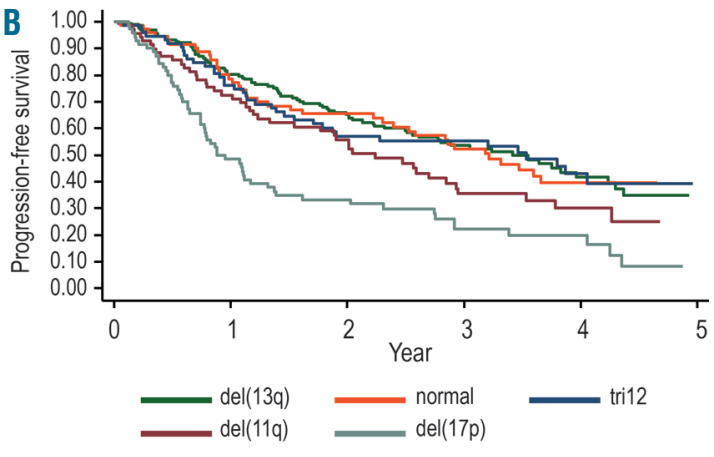

Figure 1. Progression-free survival (PFS) after first-line therapy. PFS according to (A) treatment and (B) fluorescence in situ hybridization (FISH) cytogenetic status. F/FC: fludarabine/fludarabine in combination with cyclophosphamide; CLB+/-R: chlorambucil and rituximab; FCR: fludarabine in combination with cyclophosphamide and rituximab; B/BR: bendamustine/bendamustin and rituximab; ALEM: alemtuzumab; CHOP/CVP+/-R: cyclophosphamide+hydroxydaunorubicin+vincristine+prednisone/cyclophosphamid+vincristine+prednisone+rituximab. 
this analysis showed a prognostic impact of IGHV status both on $O S(P<0.01)$ and PFS $(P=0.02)$ (data not shown).

In univariate analysis, PFS and $O S$ were also associated with type of hospital ( $P=0.05$ and $P=0.04$ ) (Figure 2C), adherence to treatment guidelines $(P=0.001$ and $P=0.006)$, and Rai stage $(P=0.042$ and $P=0.005)$. Median PFS and OS at university/regional hospitals versus other hospitals were 28 versus 22 months and 61 versus 54 months, respectively. Those treated according to national guidelines showed a median PFS and OS of 26 months and 58 months, respectively. The correlating time for those treated outside trials or guidelines were 13 months and 44 months, respectively and for those in clinical trials 19 months and 66 months, respectively. Patients on medication with ASA or statins also showed a significantly shorter PFS and OS $(P<0.001$ and $P=0.007)$ (by univariate analysis only).

\section{Safety}

Infections of grade III or higher were significantly associated with type of treatment, affecting $19 \%$ of the CLBtreated patients and $30 \%$ in the FCR group $(P=0.006)$ (Table 2). Richter transformation occurred in $5.5 \%$ of the patients, was significantly associated with $\operatorname{del}(17 \mathrm{p})$ $(P=0.04)$, and was equally distributed between types of first-line therapy. The median time to transformation was three years from diagnosis and 1.5 years from first-line treatment. Secondary malignancies affected $15 \%$ of the patients and were equally distributed between types of first-line therapy. About one-third of the secondary malignancies consisted of basal cell carcinomas. MDS/AML affected only $1 \%$ of the patients and the other secondary malignancies were solid tumors.

\section{Discussion}

Randomized controlled trials (RCTs) remain the scientific ideal for evaluation of novel treatments. However, in studies on malignancies, RCTs are sometimes not sufficient to address the evidentiary requirements of regulating authorities ${ }^{30}$ and payers as patients are selected on strict inclusion/exclusion criteria and sufficient data on overall survival and long-term follow up is often not provided. ${ }^{31}$ In addition, the comparative arm in clinical trials may be chosen to favor the treatment of investigation. Therefore, regulating authorities increasingly look for real-world data for additional comparison when evaluating new cost-intensive drug regimens. However, reliable data on consecutive patients in routine health-care may be difficult to obtain.

This is the largest retrospective cohort of strictly consecutive real-world patients from a well defined geographical region (Sweden) with a comparatively long complete follow up. By using high-quality Swedish data bases (National Cancer Registry/Swedish CLL-registry) including all patients diagnosed in Sweden within a specified time period, followed by in-depth analysis of each individual medical file, we were able to obtain a complete record of all patients diagnosed with and receiving first-
A

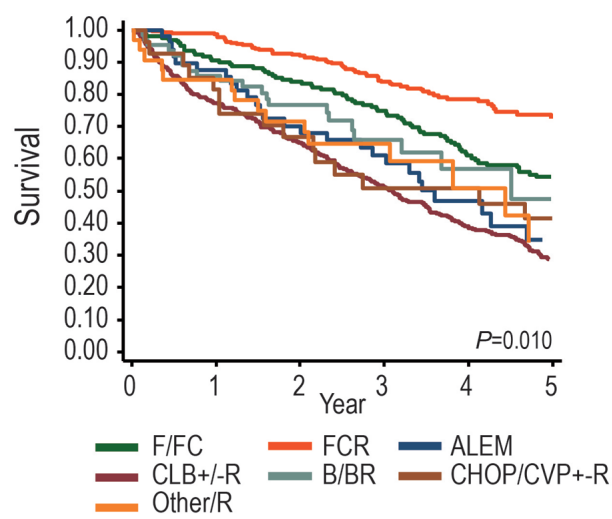

C

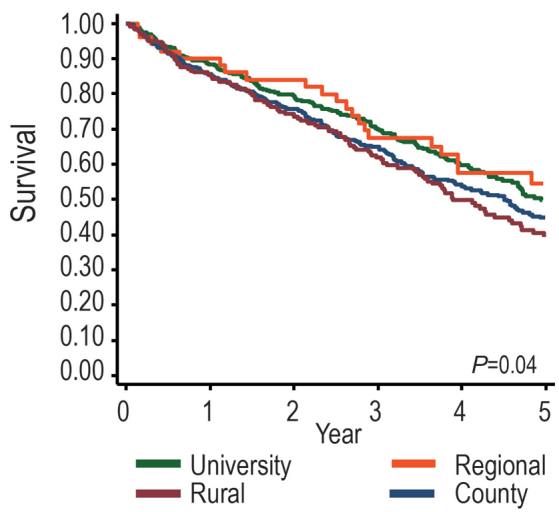

B

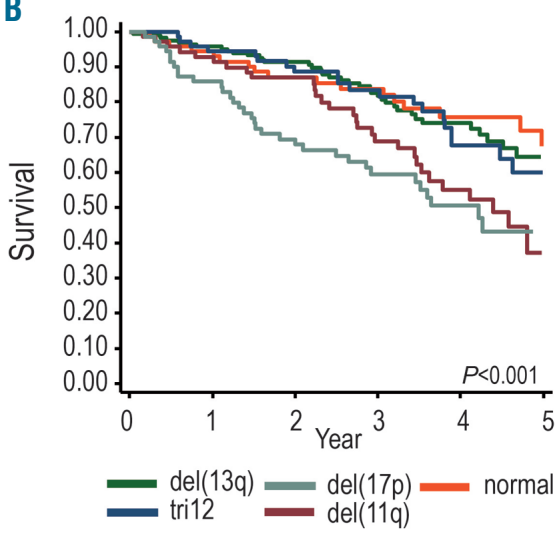

D

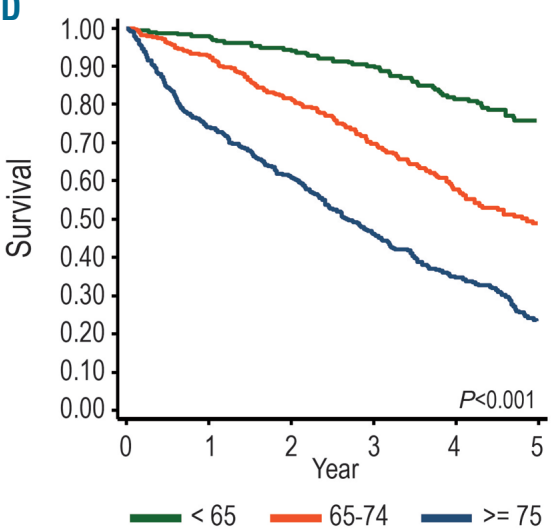

Figure 2. Overall survival (OS) after first-line therapy. OS according to (A) treatment, (B) fluorescence in situ hybridization cytogenetic status, (C) type of hospital, and (D) age. F/FC: fludarabine/fludarabine in combination with cyclophosphamide; $\mathrm{CLB}+/-$ R: chlorambucil and rituximab; FCR: fludarabine in combination with cyclophosphamide and rituximab; B/BR: bendamustine/bendamustin and rituximab; ALEM: alemtuzumab; CHOP/CVP+/-R: cyclophosphamide + hydroxydaunorubicin+vincristine $+p$ rednisone/cyclophosphamid+vincristine+prednisone+rituximab. 
line CLL treatment in the period 2007-2013. As officebased private medicine is practically non-existent for CLL in Sweden, all patient files were identified, ensuring highquality data and minimal selection bias, which is a key strength of our report. The time period 2007-2013 was selected in order to obtain sufficient follow up.

Subjects in this study were older than in previous clini- cal studies, ${ }^{6,8,32,33}$ more often had advanced disease but were in good performance status. The median age in our cohort is consistent with median age at diagnosis, ${ }^{3}$ and the advanced stage combined with good performance status reflects treatment indication and first-line treatment status. The majority of patients were treated in the later time period which possibly suggests that most patients

Table 3. Multivariate analysis* on factors in relation to progression-free survival and overall survival.

\begin{tabular}{|c|c|c|c|c|}
\hline $\begin{array}{l}\text { Clinical factor } \\
\text { (percentage available data) }\end{array}$ & $\begin{array}{c}\text { Progression-free survival } \\
\text { HR (CI 95\%) }\end{array}$ & $\mathbf{P}$ & $\begin{array}{l}\text { Overall survival } \\
\text { HR (CI 95\%) }\end{array}$ & $\mathbf{P}$ \\
\hline \multicolumn{5}{|l|}{ Age, years (100) } \\
\hline$<65$ & 1 & & 1 & \\
\hline $65-74$ & $1.41(1.02-1.93)$ & & $2.30(1.39-3.79)$ & \\
\hline$>75$ & $1.86(1.24-2.80)$ & 0.010 & $3.76(2.12-6.67)$ & $<0.001$ \\
\hline \multicolumn{5}{|l|}{ Gender (100) } \\
\hline Males & 1 & & 1 & \\
\hline Females & $0.70(0.53-0.94)$ & 0.017 & $0.60(0.40-0.91)$ & 0.016 \\
\hline \multicolumn{5}{|l|}{ Performance status (ECOG) (98) } \\
\hline 0 & 1 & & 1 & \\
\hline 1 & $1.60(1.18-2.18)$ & & $2.33(1.48-3.66)$ & \\
\hline $2-3$ & $1.87(1.12-3.13)$ & 0.006 & $2.25(1.10-4.59)$ & 0.001 \\
\hline \multicolumn{5}{|l|}{ Rai stage (98) } \\
\hline $0-2$ & 1 & & 1 & \\
\hline $3-4$ & $0.92(0.71-1.19)$ & 0.53 & $1.22(0.86-1.75)$ & 0.263 \\
\hline \multicolumn{5}{|l|}{ FISH (64) } \\
\hline $\operatorname{del}(13 q)$, normal, tri12 or del(11q) & 1 & & 1 & \\
\hline $\operatorname{del}(17 p)$ & $2.05(1.43-2.91)$ & $<0.001$ & $2.17(1.38-3.39)$ & $<0.001$ \\
\hline \multicolumn{5}{|l|}{ Treatment (99) } \\
\hline $\mathrm{F} / \mathrm{FC} / \mathrm{FCR}$ & 1 & & 1 & \\
\hline Alemtuzumab/CHOP/CVP & $1.68(1.09-2.57)$ & & $1.32(0.74-2.37)$ & \\
\hline CLB & $2.82(1.86-4.26)$ & & $2.40(1.43-4.04)$ & \\
\hline $\mathrm{B} / \mathrm{BR}$ & $0.79(0.47-1.33)$ & $<0.001$ & $1.14(0.56-2.33)$ & 0.010 \\
\hline
\end{tabular}

Hospital (100)

County/Rural

1

1

University/Regional

Region (100)

Stockholm/Gotland

$0.82(0.61-1.09)$

0.166
Uppsala/Örebro

1

Southeast

South

West

North

Treatment according to: (99)

National Guidelines - Yes

Clinical trial - Yes

National Guidelines/Clinical trial - No

Time to treatment (100)

$<$ year

$\geq 1$ year

$0.78(0.51-1.19)$

$1.03(0.65-1.63)$

$1.22(0.78-1.92)$

$0.97(0.59-1.60)$

0.93 (0.54-1.62)
0.936

HR: hazard ratio; CI: confidence interval; ECOG: Eastern Co-operative Oncology Group; FISH: fluorescence in situ hybridization; F: fludarabine; FC: fludarabine in combination with cyclophosphamide; FCR: fludarabine in combination with cyclophosphamide and rituximab; alemtuzumab/CHOP/CVP: alemtuzumab /cyclophosphamide+hydroxydaunorubicin+vincristine+prednisone/cyclophosphamide+vincristine+prednisone; CLB: chlorambucil; B/BR: bendamustine/bendamustin and rituximab. *Model included all listed factors. 
with CLL do not have a treatment indication at the time of diagnosis. Cytogenetic status was only available in $58 \%$ of the patients. The analysis was not mandatory until 2010 according to the national guidelines while FISH was more commonly performed in the later time period (2010-2013). Notably, university hospitals performed FISH significantly more often, and the older the patient the more rarely was the analysis performed.

The vast majority $(80 \%)$ were treated according to the national guidelines. Notably, compliance to national guidelines was associated with better response, and PFS and OS (univariate analysis). However, patients included in clinical trials had an even better ORR and OS but a shorter PFS. This may partly be due to our conservative approach on how to interpret response, i.e. if not all variables for a complete or partial response where available responses were considered to be of a lower grade. As patients in studies may have been more thoroughly evaluated, variables for response were possibly more available; this may have resulted in a higher response rate for these patients. Also, selection bias or more effective regimens and a more thorough follow up in clinical trials (which may detect progression at an earlier stage) may have influenced the results.

The different types of treatments used may at least partly reflect the development of therapies over time. In fact, FCR was used much more in the later time period and $\mathrm{B} / \mathrm{BR}$, which was not introduced until the end of the study period, was used less. Even though CLB use declined during the study period, approximately onethird of all patients still received first-line CLB as late as 2013, although with significant regional differences. Regarding FCR and BR, the response rate was slightly lower than in prospective first-line clinical studies ${ }^{6,8,9}$ and for CLB it was slightly higher. ${ }^{10,34}$ As expected, and in line with recently published data,, 32 FCR was more commonly used in younger patients and CLB was the most frequently used treatment overall and in the elderly. The median age for CLB-treated patients did not increase over time, possibly indicating that more modern treatments, such as B/BR, were not always used in patients over 65 years of age. OS differed between the two time periods (2007-2009 vs. 2010-2013), but this difference was not significant. This is in line with our previous findings regarding second-line therapy in the Stockholm region, ${ }^{1}$ where no improvement in OS over the years was seen.

Real-world treatment outcome may differ from that in clinical trials and when compared to data from prospective trials, ${ }^{6,8,9}$ patients in this study showed a relatively low ORR and short PFS and OS, despite an $80 \%$ compliance to national guidelines.

In line with previous data, $\operatorname{del}(17 \mathrm{p})^{14-16}$ was a negative predictive marker but also type of treatment, age and performance status were independently associated with poorer PFS and OS. Patients in this report were comparatively old versus those in trials, ${ }^{6,8,32}$ and as many as $39 \%$ received CLB and CLB treatment was associated with poorer outcome. In addition, cytogenetic status, the strongest predictor of outcome, was unknown in almost half the patients. These factors may at least partly explain the differences in our results and those reported in trials. ${ }^{6,8,32}$ Notably, patients treated with single CLB in clinical trials ${ }^{34-36}$ showed an even shorter PFS; this may be due to a more thorough evaluation in trials. Also, patients treated with B/BR showed a comparatively poor out- come. ${ }^{8}$ However, these regimens were used only in the elderly, and only FCR and FC showed better outcomes. The difference in OS between the time periods was not significant $(P=0.07)$, and this may possibly be influenced by the low use of FCR and by the fact that bendamustine became available only at the end of the time period. IGHV mutation status is still optional in the Swedish guidelines, probably explaining the low number of patients analyzed. Thus, the power of the analysis was insufficient for the multivariate analysis. However, our findings for the whole population was in line with previous reports ${ }^{37}$ in showing a significant impact on outcome.

The infection rate re, $^{6,10,34}$ was comparable to previous clinical studies with an expected higher infection rate for FCR. Our study showed an incidence of RT in line with previous data. ${ }^{32,38-40}$ However, our study did not, in contrast to previous data, ${ }^{38,41}$ show any association between treatment with fludarabine and RT. The slightly lower incidence of RT within the CLB group may be because the median time to transformation was three years, and the patients in this group were older and had a shorter median OS.

Our study indicates that type of hospital may possibly have an impact on outcome but could not confirm previous findings ${ }^{23}$ regarding outcome in urban versus rural regions. The possible differences between type of hospitals may derive from the fact that FISH analysis was more often performed at university hospitals, and the widespread use of the regularly up-dated Swedish National Guidelines may have minimized the difference between regions as $80 \%$ were treated according to guidelines. The regional usage of chlorambucil varied between $27-49 \%$. We still have no explanation for this. Elderly patients and those with a poorer performance status might also be on concomitant medication with ASA or statins, i.e. those with certain comorbidity. This may explain why concomitant ASA or statins showed a significant association in univariate but not in multivariate analysis. ${ }^{42}$ Also, in some previous reports, ASA or statins do not appear to affect outcome. ${ }^{43,44}$

A limitation of the study is its retrospective nature and the lack of data from recent years during which different regimens have been used and novel therapies have become available. For example, part of the study was performed before bendamustine was introduced as first-line treatment in CLL and before a CD20 antibody was added to CLB. For a more complete understanding of real-world outcome in CLL patients, an analysis of the outcome of treatment of relapse is warranted. As this requires a longer follow up, we have started a separate project for further investigation. Despite these limitations, in relation to today's standard-of-care treatment, the results are still important.

In summary, our results provide additional information representative of real-world outcome of first-line CLL treatment and provide an important context within which to evaluate the findings obtained from clinical trials of new drugs. We show that outcome in real-world situations differs from that in clinical trials, and that single-agent CLB treatment, as well as age and performance status, were independent factors for poor outcome in multivariate analysis. Notably, the older the patient the more rarely was FISH analysis performed and the more often CLB was chosen as treatment. As CLL and related complications seem to be the major cause of death in 
patients, regardless of comorbidity ${ }^{45,46}$ also elderly, comorbid patients should preferably undergo cytogenetic analysis and receive treatments for adequate disease control. Hence, we conclude that alternative modern, effective first-line treatment alternatives must be offered to elderly comorbid patients. Our study also raises the question as to whether drugs other than CLB, even if combined with a CD20 antibody, ${ }^{47}$ should be considered as the chemotherapy approach in the standard-of-care arm in pivotal clinical trials. Finally, we have also demonstrated inter-regional differences in drug and FISH usage, and that outcome may vary in different parts of the country despite regular updates on generally available national
CLL guidelines; findings which need to be further investigated in detail.

\section{Funding}

This study was a collaboration within the Swedish CLL group and was supported by grants from AFA Insurance (Ref no: 130054), SLL/ALF (Ref no: 20150070), Blodcancerfonden 2016, Dr Ảke Olsson Foundation (Ref no: 2-791/2016), SLL/KI Högre klinisk forskare 2018/2019 (K2894-2016), Svenska Läkaresällskapet (SLS-406961), The Gilead Sciences Nordic Fellowship Programme 2015 (LH), The Swedish Cancer Society (Ref no: 150930, 160534). We thank Ms Leila Relander for editorial assistance.

\section{References}

1. Asklid A, Winqvist M, Eketorp Sylvan S, et al. Outcomes of second-line treatment in chronic lymphocytic leukemia - a population-based study from a well defined geographical region between 2003 and 2013 . Leuk Lymphoma. 2017;58(5):1219-1223.

2. Eichhorst B, Dreyling M, Robak T, et al. Chronic lymphocytic leukemia: ESMO Clinical Practice Guidelines for diagnosis, treatment and follow-up. Ann Oncol. 2011;22(Suppl 6):vi50-54

3. Svenska KLL gruppen. https://www.cancercentrum.se/syd/cancerdiagnoser/blod-lymfom-myelom/kronisk-lymfatisk-leukemi$\mathrm{kll} /$ vardprogram/. Regionalt cancercentrum Stockholm och Gotland. 2016

4. Siegel RL, Sahar L, Portier KM, Ward EM, Jemal A. Cancer death rates in US congressional districts. CA Cancer J Clin. 2015;65(5):339-344.

5. Hallek M, Cheson BD, Catovsky D, et al. Guidelines for diagnosis, indications for treatment, response assessment and supportive management of chronic lymphocytic leukemia. Blood. 2018;131(25):2745-2760.

6. Hallek M, Fischer K, Fingerle-Rowson G, et al. Addition of rituximab to fludarabine and cyclophosphamide in patients with chronic lymphocytic leukaemia: a randomised, open-label, phase 3 trial. Lancet. 2010; 376(9747):1164-1174

7. Cramer P, Fink AM, Busch R, et al. Secondline therapies of patients initially treated with fludarabine and cyclophosphamide or fludarabine, cyclophosphamide and rituximab for chronic lymphocytic leukemia within the CLL8 protocol of the German CLL Study Group. Leuk Lymphoma. 2013; 54(8):1821-1822.

8. Eichhorst B, Fink AM, Bahlo J, et al. First-line chemoimmunotherapy with bendamustine and rituximab versus fludarabine, cyclophosphamide, and rituximab in patients with advanced chronic lymphocytic leukaemia (CLL10): an international, openlabel, randomised, phase 3, non-inferiority trial. Lancet Oncol. 2016;17(7):928-942.

9. Fischer K, Cramer P, Busch R, et al. Bendamustine in combination with rituximab for previously untreated patients with chronic lymphocytic leukemia: a multicenter phase II trial of the German Chronic Lymphocytic Leukemia Study Group. J Clin Oncol. 2012;30(26):3209-3216.

10. Goede V, Fischer K, Busch R, et al.
Obinutuzumab plus chlorambucil in patients with CLL and coexisting conditions. N Engl J Med. 2014;370(12):1101 1110

11. Hillmen P, Robak $T$, Janssens A, et al. Chlorambucil plus ofatumumab versus chlorambucil alone in previously untreated patients with chronic lymphocytic leukaemia (COMPLEMENT 1): a randomised, multicentre, open-label phase 3 trial. Lancet. 2015;385(9980):1873-1883.

12. Michallet AS, Aktan M, Hiddemann W, et al. Rituximab plus bendamustine or chlorambucil for chronic lymphocytic leukemia: primary analysis of the randomized, openlabel MABLE study. Haematologica. 2018;103(4):698-706.

13. Stilgenbauer S, Schnaiter A, Paschka P, et al. Gene mutations and treatment outcome in chronic lymphocytic leukemia: results from the CLL8 trial. Blood. 2014;123(21):32473254

14. Zenz T, Eichhorst B, Busch R, et al. TP53 mutation and survival in chronic lymphocytic leukemia. J Clin Oncol. 2010; 28(29):4473-4479

15. Dohner $\mathrm{H}$, Stilgenbauer $\mathrm{S}$, Benner A, et al Genomic aberrations and survival in chronic lymphocytic leukemia. N Engl J Med. 2000;343(26):1910-1916.

16. Rossi D, Rasi S, Spina V, et al. Integrated mutational and cytogenetic analysis identifies new prognostic subgroups in chronic lymphocytic leukemia. Blood. 2013; 121(8):1403-1412

17. Byrd JC, Brown JR, O'Brien S, et al. Ibrutinib versus ofatumumab in previously treated chronic lymphoid leukemia. N Engl J Med. 2014:371(3):213-223.

18. Byrd JC, Furman RR, Coutre SE, et al. Targeting BTK with ibrutinib in relapsed chronic lymphocytic leukemia. N Engl Med. 2013;369(1):32-42.

19. Furman RR, Cheng $S$, Lu $P$, et al. Ibrutinib resistance in chronic lymphocytic leukemia. N Engl J Med. 2014; 370(24):2352-2354.

20. Jones J, Mato A, Coutre S, et al. Evaluation of 230 patients with relapsed/refractory deletion $17 p$ chronic lymphocytic leukaemia treated with ibrutinib from 3 clinical trials. Br J Haematol 2018; 182(4):504-512.

21. O'Brien S, Jones JA, Coutre SE, et al Ibrutinib for patients with relapsed or refractory chronic lymphocytic leukaemia with $17 \mathrm{p}$ deletion (RESONATE-17): a phase 2 , open-label, multicentre study. Lancet Oncol. 2016;17(10):1409-1418
22. Lewis J, Kerridge I, Lipworth W. Use of RealWorld Data for the Research, Development, and Evaluation of Oncology Precision Medicines. JCO Precision Oncology. 2017:111.

23. Loberiza FR Ir, Cannon AJ, Weisenburger $\mathrm{DD}$, et al. Survival disparities in patients with lymphoma according to place of residence and treatment provider: a populationbased study. J Clin Oncol. 2009; 27(32):5376-5382.

24. The World Medical Association. 64th WMA General Assembly Fortaleza Brazil, October 2013. WMA Declaration of Helsinki Ethical Principles for Medical Research Involving Human Subjects. 2018 [cited; Available from: https://www.wma.net/policies-post/wma-declaration-of-helsinki-ethical-principles-for-medical-research-involving-human-subjects/

25. Bellosillo B, Pique M, Barragan M, et al Aspirin and salicylate induce apoptosis and activation of caspases in B-cell chronic lymphocytic leukemia cells. Blood. 1998; 92(4):1406-1414.

26. Chapman-Shimshoni D, Yuklea M, Radnay J, Shapiro H, Lishner M. Simvastatin induces apoptosis of B-CLL cells by activation of mitochondrial caspase 9. Exp Hematol 2003;31(9):779-783

27. Chae YK, Trinh L, Jain P, et al. Statin and aspirin use is associated with improved outcome of FCR therapy in relapsed/refractory chronic lymphocytic leukemia. Blood. 2014;123(9):1424-1426.

28. Hallek M, Cheson BD, Catovsky D, et al. Guidelines for the diagnosis and treatment of chronic lymphocytic leukemia: a report from the International Workshop on Chronic Lymphocytic Leukemia updating the National Cancer Institute-Working Group 1996 guidelines. Blood. 2008 111(12):5446-5456.

29. Socialstyrelsen. http://www.social styrelsen.se/register/dodsorsaksregistret.

30. Lewis J, Kerridge I, Lipworth W. Use of realworld data for the research, revelopment, and evaluation of oncology precision medicines. JCO Precision Oncology. 2017:1-11

31. Lewis JR, Lipworth WL, Kerridge IH, Day RO. The economic evaluation of personalised oncology medicines: ethical challenges. Med J Aust. 2013;199(7):471-473

32. Huang SJ, Lee LJ, Gerrie AS, et al. Characterization of treatment and outcomes in a population-based cohort of patients with chronic lymphocytic leukemia referred 
for cytogenetic testing in British Columbia, Canada. Leuk Res. 2017;55:79-90.

33. Tam CS, O'Brien S, Plunkett W, et al. Longterm results of first salvage treatment in CLL patients treated initially with FCR (fludarabine, cyclophosphamide, rituximab). Blood. 2014;124(20):3059-3064.

34. Burger JA, Tedeschi A, Barr PM, et al. Ibrutinib as Initial Therapy for Patients with Chronic Lymphocytic Leukemia. N Engl J Med. 2015;373(25):2425-2437.

35. Eichhorst BF, Busch R, Stilgenbauer S, et al. First-line therapy with fludarabine compared with chlorambucil does not result in a major benefit for elderly patients with advanced chronic lymphocytic leukemia. Blood. 2009:114(16):3382-3391

36. Hillmen P, Skotnicki AB, Robak T, et al. Alemtuzumab compared with chlorambucil as first-line therapy for chronic lymphocytic leukemia. J Clin Oncol. 2007; 25(35):56165623.

37. Gentile M, Shanafelt TD, Mauro FR, et al. Comparison between the CLL-IPI and the Barcelona-Brno prognostic model: Analysis of 1299 newly diagnosed cases. Am J Hematol. 2018;93(2):E35-E37.

38. Benjamini O, Jain P, Trinh L, et al. Second cancers in patients with chronic lymphocytic leukemia who received frontline fludarabine, cyclophosphamide and rituximab therapy: distribution and clinical outcomes. Leuk Lymphoma. 2015:56(6):1643-1650.

39. Tam CS, O'Brien S, Wierda W, et al. Longterm results of the fludarabine, cyclophosphamide, and rituximab regimen as initial therapy of chronic lymphocytic leukemia. Blood. 2008;112(4):975-980.

40. Woyach JA, Ruppert AS, Heerema NA, et al. Chemoimmunotherapy with fludarabine and rituximab produces extended overall survival and progression-free survival in chronic lymphocytic leukemia: long-term follow-up of CALGB study 9712. J Clin Oncol. 2011;29(10):1349-1355.

41. Fischer K, Bahlo J, Fink AM, et al. Long-term remissions after FCR chemoimmunotherapy in previously untreated patients with CLL: updated results of the CLL8 trial. Blood. 2016;127(2):208-215.

42. Thurmes P, Call T, Slager S, et al. Comorbid conditions and survival in unselected, newly diagnosed patients with chronic lymphocytic leukemia. Leuk Lymphoma. 2008;49(1): 49-56.

43. Friedman DR, Magura LA, Warren HA, et al.
Statin use and need for therapy in chronic lymphocytic leukemia. Leuk Lymphoma. 2010;51(12):2295-2298.

44. Shanafelt TD, Rabe KG, Kay NE, et al. Statin and non-steroidal anti-inflammatory drug use in relation to clinical outcome among patients with Rai stage 0 chronic lymphocytic leukemia. Leuk Lymphoma. 2010;51(7):1233-1240

45. Goede V, Cramer P, Busch R, et al Interactions between comorbidity and treatment of chronic lymphocytic leukemia: results of German Chronic Lymphocytic Leukemia Study Group trials. Haematologica. 2014;99(6):1095-1100.

46. Strati P, Parikh SA, Chaffee KG, et al Relationship between co-morbidities at diagnosis, survival and ultimate cause of death in patients with chronic lymphocytic leukaemia (CLL): a prospective cohort study. Br J Haematol. 2017;178(3):394-402.

47. Hillmen P, Janssens A, Babu KG, et al. Healthrelated quality of life and patient-reported outcomes of ofatumumab plus chlorambucil versus chlorambucil monotherapy in the COMPLEMENT 1 trial of patients with previously untreated CLL. Acta Oncol. 2016;55(9-10):1115-1120. 